\title{
Fractional calculus as a macroscopic manifestation of randomness
}

\author{
P. Grigolini, ${ }^{1,2,3}$ A. Rocco, ${ }^{1}$ and B. J. West ${ }^{1}$ \\ ${ }^{1}$ Center for Nonlinear Science, University of North Texas, P.O. Box 5368, Denton, Texas 76203-5368 \\ ${ }^{2}$ Dipartimento di Fisica dell'Università di Pisa, Piazza Torricelli 2, 56100 Pisa, Italy \\ ${ }^{3}$ Istituto di Biofisica del Consiglio Nazionale delle Ricerche, Via S. Lorenzo 26, 56127 Pisa, Italy
}

(Received 6 August 1998)

\begin{abstract}
We generalize the method of Van Hove [Physica (Amsterdam) 21, 517 (1955)] so as to deal with the case of nonordinary statistical mechanics, that being phenomena with no time-scale separation. We show that in the case of ordinary statistical mechanics, even if the adoption of the Van Hove method imposes randomness upon Hamiltonian dynamics, the resulting statistical process is described using normal calculus techniques. On the other hand, in the case where there is no time-scale separation, this generalized version of Van Hove's method not only imposes randomness upon the microscopic dynamics, but it also transmits randomness to the macroscopic level. As a result, the correct description of macroscopic dynamics has to be expressed in terms of the fractional calculus. [S1063-651X(99)11802-6]

PACS number(s): 05.40.-a, 05.45.-a, 05.60.-k
\end{abstract}

\section{INTRODUCTION}

The physical paradigm of statistical physics is Brownian motion, which involves diffusion, dissipation, and the fluctuation-dissipation relation tying the two together. The dynamical model of this process was provided by Langevin in 1908 using a stochastic differential equation. In spite of this long history, it seems apparent from the nature of randomness that such macroscopic stochastic equations are incompatible with the continuous and differentiable character of microscopic Hamiltonian dynamics. However, it is widely believed that Brownian motion can be rigorously derived from the totally deterministic Hamiltonian models of classical mechanics. Part of the reason for this conviction has to do with the wide use made in literature of Van Hove's method [1-3]. In one form or another, many of the attempts currently made to establish a unified view of mechanics and thermodynamics [4] can be traced back to the method of Van Hove. The result of this method depends on whether we adopt the Heisenberg perspective, corresponding to the time evolution of observables, or the Schrödinger perspective, corresponding to the time evolution of the Liouville density. In the former case, the usual outcome is the derivation from mechanics of an ordinary Langevin equation. In the latter case, the adoption of the Van Hove method yields master equations. We focus here especially on the conventional diffusion equation, with the diffusion process described by a second-order spatial derivative.

In the Heisenberg perspective, after averaging over an ensemble of realizations of the stochastic force, the relaxation process is described by an exponential function. In the Schrödinger perspective, the mathematical representation of the diffusion process is given, as we have said, by a secondorder spatial derivative of a density function. Therefore, the mathematical description rests on either ordinary analytical functions (exponential functions) describing the dynamics, or on conventional differential operators (second-order derivatives) describing the phase space evolution. This is probably the reason why there is no mention of the fact, and indeed no perception, that the Van Hove method is equivalent to intro- ducing stochastic dynamics, namely, discrete processes and unpredictable nondifferentiable jumps, in the dominion of continuous and differentiable Hamiltonian dynamics.

Actually the differentiable nature of the macroscopic picture is, in a sense, a natural consequence of the microscopic randomness [5,6], and of the related nondifferentiability as well, due to the key role of the central limit theorem. Recall that in the central limit theorem the quantities being added together are statistically independent, or at most weakly dependent, in order for the theorem to be applicable and Gaussian statistics to emerge. Once a condition of time-scale separation is established, in the long-time limit the memory of the nondifferentiable character of microscopic dynamics is lost, and Gaussian statistics result. This also means that use can be made again of ordinary differential calculations on the macroscopic scale, even if the microscopic dynamics are incompatible with the adoption of ordinary calculus methods.

On the other hand, in the case where a time-scale separation between macroscopic and microscopic levels of description does not exist, the nondifferentiable nature of the microscopic dynamics is transmitted to the macroscopic level. An illuminating example is given by the paper of Ref. [7], which shows that a diffusion process generated by a fluctuation with no time scale at the macroscopic level generates a diffusion process well described by a fractional Laplacian $[8,9]$. The paper of Ref. [7] also addresses the intriguing problem of making a Lévy process, which has an infinite second moment, compatible with the dynamical approach to diffusion. The dynamical approach to diffusion rests on steps of finite length, and consequently results in finite second moments. This problem has been addressed in a variety of ways $[10,11]$, ranging from taking into account the finite size $L$ of the sample, within which the Lévy flight takes place [10], to the case where the probability density is truncated [11]. We rather follow the prescriptions of Ref. [7], which is a form of Lévy walk, where the individual jumps are not instantaneous and involve a time cost, thereby making it possible for us to adopt a diffusion picture with finite moments. All this was discussed in Ref. [7], and here the results of Ref. [7] are made compatible with the infinite moments of the Lévy sta- 
tistics by means of the generalized Van Hove method. Another case where the adoption of a fractional calculus is made necessary concerns the generalization of the exponential form of relaxation according to the recent prescriptions of Nonnenmacher and co-workers [12,13], involving the concept of a fractional time derivative. The main aim of this paper is to prove that the fractional derivative, both the space fractional derivative of Ref. [7] and the time fractional derivatives of Refs. [12,13] are made compatible with Hamiltonian deterministic dynamics by means of a generalized version of Van Hove's method.

In fact, if we make the traditional classical assumption that the microscopic dynamics follow the Newton prescription, we also have to address the problem of how to make this prescription compatible with randomness. The solution to this problem requires that an extension of the Van Hove method be discovered. This naturally yields the working hypothesis that the fractional derivatives, currently used to describe macroscopic transport processes $[8,12,13]$, can be regarded as the macroscopic manifestation, in the absence of time-scale separation, of either nondifferentiable microscopic dynamics, an assumption that would violate the applicability of Hamiltonian dynamics to this domain, or of a Hamiltonian description that loses differentiability through a kind of filtering described by a generalized Van Hove approximation. In other words, just as the Van Hove method makes Brownian motion compatible with Hamiltonian dynamics, a generalized Van Hove method is used here to make the macroscopic fractional calculus compatible with microscopic Hamiltonian dynamics. The main difference from the case of ordinary statistical mechanics is that in this nonordinary case the nondifferentiable nature of microscopic dynamics, either natural or forced by the adoption of the Van Hove prescription, is transmitted to the macroscopic level where it takes the shape of fractional derivatives. The main purpose of this paper is to substantiate this working assumption with convincing arguments.

The outline of the paper is as follows. In Sec. II we express the Van Hove method in a form equivalent to the ordinary method, but more convenient for the generalization that we plan to develop in this paper. In Sec. III we show that in the Heisenberg picture the generalization of the Van Hove method results in the fractional derivatives currently used by Nonnenmacher and co-workers to study polymer dynamics. Section IV and Appendix A are devoted to the Schrödinger picture, and show that the Van Hove method in this picture leads to the fractional derivative introduced by Seshadri and West [8] (see also West and Grigolini [9]) to describe Lévy processes. Section V is devoted to concluding remarks.

\section{VAN HOVE LIMIT: AN EXAMPLE FROM A SPECIFIC MODEL}

As pointed out in Sec. I, the Van Hove limit [1] of a microscopic process turns out to be a key ingredient for the derivation of statistical mechanics from microscopic dynamics. We review this limit and how it works in the case of a specific model rather than in general.

Let us start by recalling the meaning of this limit. Consider the integrodifferential equation

$$
\frac{d f(t)}{d t}=-\lambda^{2} \int_{0}^{t} K\left(t-t^{\prime}\right) f\left(t^{\prime}\right) d t^{\prime},
$$

where $f$ is some quantity of interest, $K$ is a memory kernel, and $\lambda$ is a parameter. Equation (1) is a typical nonMarkovian equation obtained in studying physical systems coupled to an environment, and whose environmental degrees of freedom have been averaged over. In this case the parameter $\lambda$ can be regarded as the strength of the perturbation induced by the environment on the system of interest.

In the literature, wide use is made of the Markov approximation [14], which replaces the integrodifferential equation (1) with the rate equation

$$
\frac{d f(t)}{d t}=-\left(\lambda^{2} \int_{0}^{\infty} K\left(t^{\prime}\right) d t^{\prime}\right) f(t) .
$$

The Van Hove limit [2] consists of making the limit $\lambda$ $\rightarrow 0, \quad t \rightarrow \infty$ in such a way that the product $\lambda^{2} t$ is kept constant. That is, setting $x=\lambda^{2} t$ and $F(x)=f(t)$, Eq. (1) becomes

$$
\frac{d F(x)}{d x}=-\int_{0}^{x / \lambda^{2}} K\left(t^{\prime}\right) F\left(x-\lambda^{2} t^{\prime}\right) d t^{\prime} .
$$

Now the adoption of the limit

$$
\text { const }=x=\lim _{\substack{\lambda \rightarrow 0 \\ t \rightarrow \infty}} \lambda^{2} t
$$

makes it possible for us to replace the time convoluted form in Eq. (1) with

$$
\frac{d f(t)}{d t}=-\lambda^{2} \tau f(t),
$$

where

$$
\tau=\int_{0}^{\infty} d t K(t)
$$

Equation (5) gives an exponential solution, the same as that obtained by means of the Markov approximation (2).

Taking the limit $\lambda \rightarrow 0$ corresponds to assuming that the coupling of the system to the environment is weak, while the limit $t \rightarrow \infty$ means that the observation time is much larger than other temporal scales present in the system. Specifically this time must be larger than the microscopic time $\tau$. This remark allows us to reformulate the Van Hove limit in a slightly different way, more suitable for our purposes. First, instead of taking the $t \rightarrow \infty$ limit, we shall take the limit $\tau$ $\rightarrow 0$. Also, we shall replace the limit $\lambda \rightarrow 0$ with the equivalent limit $V \rightarrow \infty$, where $V$ is a coupling constant, to be specified in the following. The quantity to be kept constant in carrying out the limit is just the product $V^{2} \tau$. Notice that connecting $V, \tau$, and $\lambda$ as $\lambda=V \tau$ makes it possible to keep $V \ll \tau^{-1}$ (in such a way that $\lambda \ll 1$ ), and at the same time to set $V^{2} \rightarrow \infty$ so as to ensure $V^{2} \tau \rightarrow$ const.

The meaning of Eqs. (3)-(5) can be illustrated by adopting this equivalent perspective, and can be shown to be a way of disregarding the time evolution of the system at both 
very short and very long times, where the deviations from the exponential relaxation show up [15]. In the Hamiltonian case the relaxation cannot be rigorously exponential [16], thereby preventing a satisfactory connection between microscopic dynamics and stochastic physics [17]. We now show this benefic effect of the Van Hove method with the help of an illustrative example, where the origin of Brownian motion stems from the average over an initial statistical distribution [6] rather than from chaos [5]. Consider a chain of $2 N+1$ linear harmonic oscillators all with equal spring constants, and described by the Hamiltonian

$$
H=\sum_{i=-N}^{N} \frac{p_{i}^{2}}{2 m_{i}}+\frac{k}{2} \sum_{i=-N}^{N}\left(q_{i+1}-q_{i}\right)^{2},
$$

where $m_{i}=m$ if $i \neq 0$ and $m_{0}=M$. Vitali and Grigolini [18] proved that the correlation function for the momentum $p_{0}$ of the system of interest,

$$
\Phi_{0}(t) \equiv \frac{\left\langle p_{0} p_{0}(t)\right\rangle}{\left\langle p_{0}^{2}\right\rangle},
$$

satisfies the integral equation

$$
\dot{\Phi}_{0}=-\Delta_{1}^{2} \int_{0}^{t} \Phi_{1}\left(t-t^{\prime}\right) \Phi_{0}\left(t^{\prime}\right) d t^{\prime},
$$

where

$$
\Delta_{1}^{2}=2 k / M,
$$

and $\Phi_{1}(t)$ represents the correlation function of the stochastic force in the corresponding generalized Langevin equation (see Sec. III). The parameter $\Delta_{1}$ plays the same role as that of the earlier mentioned coupling constant $V$, while $\tau$ is given in this specific model by the expression

$$
\tau \equiv \int_{0}^{\infty} \Phi_{1}(t) d t=\sqrt{\frac{m}{k}} .
$$

In the long-time region, the solution to Eq. (9) is given by [18]

$$
\begin{aligned}
\Phi_{0}(t)= & \frac{1-\mu}{1-2 \mu} \exp \left(-\frac{\mu \omega_{0} t}{\sqrt{1-2 \mu}}\right) \\
& +\frac{2 \mu}{\pi} \int_{0}^{\infty} \frac{\sin \left(x \omega_{0} t\right) \sqrt{x^{2}-1}}{(1-2 \mu) x^{2}+\mu^{2}} d x \\
\approx & \frac{1-\mu}{1-2 \mu} \exp \left(-\frac{\mu \omega_{0} t}{\sqrt{1-2 \mu}}\right) \\
& +\frac{\mu}{(1-\mu)^{2}} \sqrt{\left(\frac{2}{\pi\left(\omega_{0} t\right)^{3}}\right)} \sin \left(\omega_{0} t-\frac{\pi}{4}\right),
\end{aligned}
$$

where

$$
\mu=m / M
$$

and

$$
\omega_{0}^{2}=4 \mathrm{k} / \mathrm{m} .
$$

The asymptotic approximation (12) shows that the momentum autocorrelation function consists of the sum of an exponentially decaying term and a nonexponential oscillatory contribution that decays as an inverse power law in time. Notice that $\omega_{0}$ is a Debye-like cutoff frequency that is related to the microscopic time scale $\tau$ as $\tau=2 / \omega_{0}$. The general form of this solution had also been observed and discussed by Zwanzig [2].

Now the Van Hove limit expressed in terms of the parameters of the model becomes

$$
\gamma \equiv \lim _{\substack{\tau \rightarrow 0 \\ \Delta_{1}^{2} \rightarrow \infty}} \Delta_{1}^{2} \tau=\lim _{\substack{\mu \rightarrow 0 \\ \omega_{0} \rightarrow \infty}} \omega_{0} \mu .
$$

Notice that this limit can be realized assuming $m \rightarrow 0$ and $M \rightarrow 0$ as $m^{1 / 2}$. The closer to zero the mass $m$ is, the better the physical condition $M \gg m$ is fulfilled (the better the macroscopic description of the system of interest). Consequently, the oscillatory tails in Eq. (12) cancel, and an exact exponential relaxation is recovered:

$$
\Phi_{0}(t)=\exp (-\gamma t)
$$

The rationale for this result stays in the formal similarity of Eqs. (9) and (1). Letting, as mentioned before, $\lambda=\Delta_{1} \tau$, it is a simple matter to verify that $\lambda \rightarrow 0$ as $m^{1 / 4}$, and the ordinary Van Hove limit (4) can be applied. More in general, we can assume that relation (15) holds true independently of the model, and we can regard the parameters $\mu$ and $\omega_{0}$ as free parameters of the theory. If we adopt this view, the exponential decay is recovered by making the Van Hove limit as described by the right-hand side of Eq. (15).

In the following, we shall refer to the Van Hove limit, rather than using the known version of Eq. (4), as expressed in our nonconventional form

$$
\gamma \equiv \lim _{\substack{\tau \rightarrow 0 \\ \Delta_{1}^{2} \rightarrow \infty}} \Delta_{1}^{2} \tau \equiv \operatorname{Lim}_{\mathrm{VH}} \Delta_{1}^{2} \tau .
$$

A final remark concerns the fact that the same result [Eq. (16)] could have been obtained by applying the Markov approximation to Eq. (9). In that case, the basic assumption would have been an infinite time-scale separation between the microscopic time scale $\tau$ and the macroscopic scale defined as the inverse of the frequency $\Omega^{2}=2 k / M$; that is,

$$
T=\sqrt{\frac{M}{2 k}} .
$$

Notice that the time-scale separation between the system of interest and bath is rendered infinitely large exactly by the same limits as those used in order to carry out the Van Hove limit: $m \rightarrow 0$ and $M \rightarrow 0$ as $m^{1 / 2}$. This demonstrates that the Van Hove limit and the Markov approximation are essentially equivalent to one another. 


\section{HEISENBERG PICTURE: ORDINARY AND FRACTIONAL RELAXATION}

It is well known [19] that the generalized Langevin equation

$$
\dot{v}=-\Delta_{1}^{2} \int_{0}^{t} \Phi_{1}\left(t-t^{\prime}\right) v\left(t^{\prime}\right) d t^{\prime}+f(t)
$$

corresponds to the following hierarchy of correlation functions:

$$
\dot{\Phi}_{i}=-\Delta_{i+1}^{2} \int_{0}^{t} \Phi_{i+1}\left(t-t^{\prime}\right) \Phi_{i}\left(t^{\prime}\right) d t^{\prime} .
$$

Equation (9) is the $i=0$ case of Eq. (20).

In order to derive both normal and anomalous relaxation properties, we are interested in making a nontrivial choice of the correlation function $\Phi_{1}(t)$. However, we also want our choice to be compatible with a completely dynamical approach. Therefore, we need to identify the conditions necessary to assure that both these constraints are satisfied. First, by means of Laplace transforms and the convolution form of Eq. (20), it is easy to prove that $\Phi_{1}$ can be represented in the form of a continued fraction:

$$
\widetilde{\Phi}_{1}(z)=\frac{1}{z+\frac{\Delta_{2}^{2}}{z+\frac{\Delta_{3}^{2}}{z+\cdots}}},
$$

where $\widetilde{\Phi}_{1}(z)$ denotes the Laplace transform of $\Phi_{1}(t)$. This structure is valid for $\Phi_{1}(0)=1$, and this is a first requirement to fulfill. Then we recall that the expansion parameters $\Delta_{i}^{2}$ can be expressed in terms of the moments

$$
s_{n} \equiv \frac{\left\langle f_{1}\left|(-L)^{n}\right| f_{1}\right\rangle}{\left\langle f_{1} \mid f_{1}\right\rangle}=\Phi_{1}^{(n)}(0),
$$

where $L$ is the Liouvillian operator driving the time evolution of the Liouville density, and $\left|f_{1}\right\rangle$ is the first state in the Mori chain $[19,20]$. An elegant way of expressing the parameters $\Delta_{i}^{2}$ in terms of the moments $s_{n}$ has been established by Grigolini et al. [21]. This implies that $\Phi_{1}(t)$ must be infinitely differentiable. Finally, the symmetry properties of the Liouvillian imply that the condition

$$
s_{2 n-1}=0
$$

also has to be fulfilled.

Therefore, we decide to focus our attention on the choice

$$
\Phi_{1}(t)=\frac{T^{\beta}}{\left(T^{2}+t^{2}\right)^{\beta / 2}} .
$$

Note that the moments $s_{n}$ are nothing but the $n$th order time derivatives of $\Phi_{1}(t)$. Thus it is straightforward to prove via successive time differentiation of Eq. (24) that the odd moments vanish. This is an important mathematical property necessary to make the relaxation process compatible with
Hamiltonian dynamics. An important example of relaxation incompatible with Hamiltonian dynamics is in fact the exponential relaxation: a case exhaustively discussed by Lee in his brilliant 1983 paper [16]. This paper proves therefore the importance of fulfilling the constraints of Eq. (23) for a generalized Langevin equation to be compatible with Hamiltonian dynamics. This condition is here fulfilled by adopting the choice of Eq. (24).

\section{A. Ordinary statistical mechanics}

Ordinary statistical mechanics can be recovered from the generalized Langevin equation (19), using Eq. (24) along with the integrability condition on the power-law index,

$$
\beta>1 .
$$

The microscopic time scale $\tau$ is given in this case in terms of the parameter $T$,

$$
\tau=\int_{0}^{\infty} d t \frac{T^{\beta}}{\left(T^{2}+t^{2}\right)^{\beta / 2}}=\frac{\sqrt{\pi}}{2} \frac{\Gamma\left(\frac{\beta-1}{2}\right)}{\Gamma\left(\frac{\beta}{2}\right)} T .
$$

Therefore, the Van Hove (VH) limit in form (17) is achieved in the limit $T \rightarrow 0$,

$$
\gamma=\frac{\sqrt{\pi}}{2} \frac{\Gamma\left(\frac{\beta-1}{2}\right)}{\Gamma\left(\frac{\beta}{2}\right)} \operatorname{Lim}_{\mathrm{VH}} \Delta_{1}^{2} T .
$$

This limiting procedure results in exponential relaxation for the correlation function $\Phi_{0}(t)$, and allows us to safely interpret Eq. (19) as identical to the ordinary Langevin equation

$$
\dot{v}=-\gamma v+f(t) .
$$

This is the traditional result obtained using the Van Hove method.

\section{B. Nonordinary statistical mechanics}

In the case where the power-law index of the correlation function is in the interval

$$
0<\beta<1,
$$

the nonintegrability of the correlation function (24) prevents us from adopting the above approach. In the case $0<\beta<1$, we are forced to look for a different procedure to go from a microscopic to a macroscpic description of the system. This procedure can be derived in a natural way from the original Van Hove limit. Let us consider the limit

$$
Q=\lim _{\substack{T \rightarrow 0 \\ \Delta_{1}^{2} \rightarrow \infty}} \Delta_{1}^{2} T^{\beta} \equiv \operatorname{Lim}_{\mathrm{GVH}} \Delta_{1}^{2} T^{\beta} .
$$

We shall refer to this limit as to the generalized Van Hove limit $(\mathrm{GVH})$.

Adopting the ansatz (30), and inserting Eq. (24) into Eq. (19), we obtain 


$$
\begin{aligned}
\dot{\Phi}_{0}= & -\operatorname{Lim}_{\mathrm{GVH}} \Delta_{1}^{2} T^{\beta} \\
& \times \int_{0}^{t} \frac{1}{\left[T^{2}+\left(t-t^{\prime}\right)^{2}\right]^{\beta / 2}} \Phi_{0}\left(t^{\prime}\right) d t^{\prime} \\
= & -Q \int_{0}^{t} \frac{1}{\left(t-t^{\prime}\right)^{\beta}} \Phi_{0}\left(t^{\prime}\right) d t^{\prime} .
\end{aligned}
$$

For dimensional reasons, it is convenient to write

$$
Q \equiv V^{2} \tau^{\beta}
$$

Notice that in general the correlation function is related to the waiting time distribution $\psi(t)$ of the process under study as

$$
\Phi_{0}(t)=1-\int_{0}^{t} \psi\left(t^{\prime}\right) d t^{\prime}
$$

and therefore Eq. (31) can be rewritten

$$
\psi(t)=Q \int_{0}^{t} \frac{1}{\left(t-t^{\prime}\right)^{\beta}} \Phi_{0}\left(t^{\prime}\right) d t^{\prime}
$$

Now, we want to compare this last expression with the fractional relaxation equation obtained by Glökle and Nonnenmacher [12]. The fractional relaxation equation for a function $\Phi_{\mathrm{ML}}(t ; \nu)$ is given by

$$
\Phi_{\mathrm{ML}}(t ; \nu)-\Phi_{\mathrm{ML}}(0 ; \nu)=\frac{1}{\tau^{\nu}} \frac{d^{-\nu}}{d t^{-\nu}} \Phi_{\mathrm{ML}}(t ; \nu)
$$

where the symbol $d^{-\nu} / d t^{-\nu}$ denotes the fractional integral (see Appendix A),

$$
\frac{d^{-\nu}}{d t^{-\nu}} f(t)=\frac{1}{\Gamma(\nu)} \int_{0}^{t} \frac{f\left(t^{\prime}\right) d t^{\prime}}{\left(t-t^{\prime}\right)^{1-\nu}} .
$$

The solution of Eq. (35) is known [13] and is given by the so-called Mittag-Leffler (ML) function,

$$
\Phi_{\mathrm{ML}}(t ; \nu)=\Phi_{\mathrm{ML}}(0 ; \nu) \sum_{k=0}^{\infty} \frac{(-1)^{k}}{\Gamma(\nu k+1)}\left(\frac{t}{\tau}\right)^{\nu k},
$$

which exhibits stretched exponential behavior at short times and inverse power-law relaxation at long times.

Setting $\beta=1-\nu$, it becomes possible to compare the fractional relaxation equation with Eq. (34) to obtain

$$
\psi(t)=-Q \Gamma(1-\beta) \tau^{1-\beta}\left[\Phi_{\mathrm{ML}}(t ; 1-\beta)-\Phi_{\mathrm{ML}}(0 ; 1-\beta)\right]
$$

where the superscript $1-\beta$ corresponds to the value for $\nu$ to insert into the form of the solution (see Sec. III B). Also recalling Eq. (32), we finally obtain, for the waiting time distribution function,

$$
\psi(t)=-\left(V^{2} \tau\right) \Gamma(1-\beta)\left[\Phi_{\mathrm{ML}}(t ; 1-\beta)-\Phi_{\mathrm{ML}}(0 ; 1-\beta)\right]
$$

which maintains the interesting properties of the MittagLeffler function earlier pointed out.

It is important to stress that the resulting analytical function has been used by Glöckle and Nonnenmacher [22] to fit with a very remarkable accuracy the relaxation curves of stress experiments on glassy material. This suggests that the dynamical randomness without time-scale separation takes the shape of a time fractional derivative, and becomes experimentally detectable at the macroscopic level.

\section{SCHRÖDINGER PICTURE: GAUSSIAN AND LÉVY DIFFUSION}

Let us now consider the equation of motion for the onedimensional probability density $p(x, t)$ :

$$
\frac{\partial}{\partial t} p(x, t)=\left\langle\xi^{2}\right\rangle_{\mathrm{eq}} \int_{0}^{t} \Phi_{\xi}\left(t^{\prime}\right) \frac{\partial^{2}}{\partial x^{2}} p\left(x, t-t^{\prime}\right) d t^{\prime} .
$$

This equation refers to the process

$$
\dot{x}=\xi
$$

and $\Phi_{\xi}(t)$ is the autocorrelation function of the $\xi$ fluctuations. The integrodifferential equation of motion for the probability density is obtained from the Langevin equation by adopting the Zwanzig projection operator method in the form discussed by Grigolini [23], and it is exact under the following two conditions: (i) the dynamics of $\xi$ is independent of that of $x$, and (ii) the system producing the $\xi$ fluctuations is a two-state system.

Notice that (ii) does not necessarily mean that the variable $\xi$ is dichotomous. The case of anomalous diffusion generated by intermittent maps [24] is an illuminating example, where the change from a continuous two-state fluctuating variable to the dichotomous case does not produce significant effects on diffusion. From the point of view of our generalized Van Hove limit, however, the difference in the two conditions is essential. As we shall see in Sec. IV A, the replacement of $\xi$ with a dichotomous variable with the values $W$ and $-W$ is an essential ingredient of the generalized Van Hove method.

For reasons which will shortly become clear, it is convenient to write the probability distribution at time $t-t^{\prime}$ in terms of the probability distribution at time $t$ as

$$
p\left(x, t-t^{\prime}\right)=\int_{-\infty}^{\infty} F\left(x-x^{\prime},-t^{\prime}\right) p\left(x^{\prime}, t\right) d x^{\prime},
$$

where the function $F$ is a propagator. Equation (40), with the nontrivial choice (24), becomes 


$$
\begin{aligned}
\frac{\partial}{\partial t} p(x, t)= & \left\langle\xi^{2}\right\rangle_{\mathrm{eq}} \int_{-\infty}^{\infty} d x^{\prime} \int_{0}^{t} d t^{\prime} \\
& \times \frac{T^{\beta}}{\left(T^{2}+t^{\prime 2}\right)^{\beta / 2}} \frac{\partial^{2}}{\partial x^{2}} F\left(x-x^{\prime},-t^{\prime}\right) p\left(x^{\prime}, t\right) .
\end{aligned}
$$

We shall discuss both the case of ordinary diffusion, $\beta>1$, and the case of anomalous diffusion, $0<\beta<1$.

\section{A. Ordinary statistical mechanics}

Let us assume that the power-law index in the autocorrelation function is

$$
\beta>1
$$

and let us make the choice of a time-independent propagator

$$
F\left(x-x^{\prime},-t^{\prime}\right)=\delta\left(x-x^{\prime}\right) .
$$

Equation (43) then becomes

$$
\frac{\partial}{\partial t} p(x, t)=\left\langle\xi^{2}\right\rangle_{\text {eq }}\left\{\int_{0}^{t} d t^{\prime} \frac{T^{\beta}}{\left(T^{2}+t^{\prime 2}\right)^{\beta / 2}}\right\} \frac{\partial^{2}}{\partial x^{2}} p(x, t) .
$$

The microscopic time-scale $\tau$ of the autocorrelation function $\Phi_{\xi}(t)$, present in Eq. (46), is finite for $\beta>1$, and we can apply again the conventional Van Hove limit in the form

$$
D=\lim _{\substack{T \rightarrow 0 \\\left\langle\xi^{2}\right\rangle_{\mathrm{eq}} \rightarrow \infty}}\left\langle\xi^{2}\right\rangle_{\mathrm{eq}} T
$$

Substituting this expression into Eq. (46), we obtain the standard diffusion equation

$$
\frac{\partial}{\partial t} p(x, t)=D \frac{\partial^{2}}{\partial x^{2}} p(x, t) .
$$

Note that the case where the correlation function $\Phi_{\xi}(t)$ is exponential, although in conflict with the Hamiltonian constraints [16], assists us in further interpreting the meaning of the Van Hove limit. As shown in Appendix B, the use of an exponential correlation function reduces Eq. (40) to the telegraphic equation, whose exact solution, expressed in the proper limit, agrees with the result predicted by the procedure here described.

\section{B. Nonordinary statistical mechanics}

Let us consider now the case where the power-law index is in the interval

$$
0<\beta<1 \text {. }
$$

In this case, we define the generalized version of the Van Hove procedure as the recipe leading to the largest component of the diffusion process. Therefore, as a first step, we make the variable $\xi$ dichotomous, by assigning to it the val- ues $W$ and $-W$ with the basic condition that the actual values of the true variable are included in the interval $[-W, W]$ (see Fig. 1).

This leads us to assume, for the form of the propagator $F$,

$$
F\left(x-x^{\prime},-t^{\prime}\right)=\delta\left(W t^{\prime}-\left|x-x^{\prime}\right|\right),
$$

which is clearly time dependent. Equation (43) then becomes

$$
\begin{aligned}
\frac{\partial}{\partial t} p(x, t)= & -\frac{W^{\beta+1} T^{\beta}}{2} \int_{-\infty}^{\infty} d x^{\prime} p\left(x^{\prime}, t\right) \\
& \times \frac{\beta\left[W^{2} T^{2}+\left|x-x^{\prime}\right|^{2}\right]-\beta(\beta+2)\left|x-x^{\prime}\right|^{2}}{\left[W^{2} T^{2}+\left|x-x^{\prime}\right|^{2}\right]^{\beta / 2+2}} .
\end{aligned}
$$

It is evident that Eq. (51) differs from Eq. (43) by a correction term that disappears in the generalized Van Hove limit. The generalized Van Hove limit is defined in this case as

$$
Q=\lim _{\substack{T \rightarrow 0 \\ W \rightarrow \infty}} T^{\beta} W^{1+\beta},
$$

and, noticing that this implies $W^{2} T^{2} \rightarrow 0$ as $W^{-2 / \beta}$, Eq. (51) can be rewritten

$$
\frac{\partial}{\partial t} p(x, t)=\frac{\beta(\beta+1)}{2} Q \int_{-\infty}^{\infty} d x^{\prime} \frac{p\left(x^{\prime}, t\right)}{\left|x-x^{\prime}\right|^{\beta+2}} .
$$

This form of diffusion equation coincides with the WestSeshadri equation $[7,8]$ for a centrosymmetric Lévy process. The fact that Eq. (53) allows for a solution in the form of a Lévy process becomes apparent by taking its Fourier transform. Letting

$$
\mu=\beta+1 \Rightarrow 1<\mu<2,
$$

and using Ref. [25], for the Fourier transform of an inverse power law we obtain

$$
\mathcal{F}\left(\frac{1}{|x|^{1+\mu}} ; k\right)=\sqrt{\frac{2}{\pi}} \Gamma(-\mu)|k|^{\mu} \cos \left(-\frac{\pi \mu}{2}\right) .
$$

Therefore, making use of the convolution theorem for Fourier transforms, Eq. (53) becomes

$$
\frac{\partial}{\partial t} \phi(k, t)=-b|k|^{\mu} \phi(k, t) .
$$

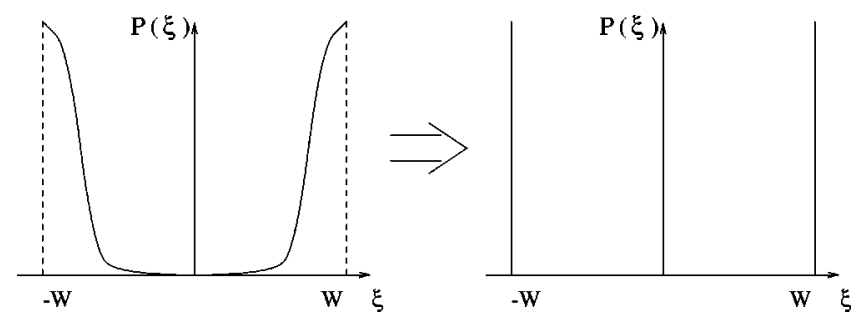

FIG. 1. Sketch of how the distribution $P(\xi)$ of the $\xi$ variable is modified so as to make the variable $\xi$ fully equivalent to a dichotomous variable. 
Here $\phi(k, t)$ is the Fourier transform of $p(x, t)$, that is, the characteristic function, and the parameter $b$ is given by

$$
b=\frac{\beta(\beta+1)}{\sqrt{2 \pi}} Q \Gamma(-\mu)\left|\cos \left(\frac{\pi \mu}{2}\right)\right|>0 .
$$

The solution to Eq. (56), with the initial condition $\phi(k, t$ $=0)=1$, necessary for the inverse Fourier transform to be defined as a probability density, is written as

$$
\phi(k, t)=e^{-b|k|^{\mu} t}
$$

which indeed corresponds to the definition of the characteristic function for a Lévy process.

Equation (53) can also be cast in the form of a fractional differential equation [9]

$$
\frac{\partial}{\partial t} p(x, t)=(-i)^{\mu} b \mathcal{D}^{(\mu)} p(x, t)
$$

where $\mathcal{D}^{(\mu)}$ is a proper definition of fractional derivative (see Appendix A). We see, in conclusion, that with the adoption of the generalized Van Hove method the standard diffusion equation yielded by the ordinary Van Hove method is expressed in terms of a fractional derivative. More in general, we expect that the ordinary Fokker-Planck equation can be replaced by a generalized expression resting on fractional derivatives [9].

In Sec. $\mathrm{V}$ we shall give more support to our conviction that the generalized form of the Van Hove method, here adopted to derive the spatial version of fractional derivatives of Eq. (59), is intimately related to the Lévy-Gnedenko theorem [26]. We limit ourselves to point out that the adoption of fractional calculus to deal with processes of anomalous diffusion is becoming more and more popular, and for the interested reader we quote Refs. [27,28], whose results, however, must be compared to the conclusion of Ref. [29] as well as to those of this section with some caution [30].

\section{CONCLUDING REMARKS}

The adoption of the Van Hove limit is essentially a sophisticated way of making the Markov approximation. The Markov approximation, in turn, establishes the physical condition necessary to make Hamiltonian dynamics compatible with stochastic physics. However, when this method is applied to microscopic dynamics to derive ordinary statistical mechanics, there is no clear perception of establishing dynamic properties inconsistent with Hamiltonian dynamics. This is so because, even if the correlation functions are made exponential by forcing the Markov approximation into microscopic dynamics, so as to become incompatible with both classical [16] and quantum [15] mechanics, they are still differentiable functions.

From the point of view of a single trajectory, the realization of the Brownian condition implies, from a rigorous mathematical point of view, the breakdown of the condition of differentiability. However, even in this case the perception of a conflict with Hamiltonian dynamics is blurred by the adoption of a statistical perspective. Within the Schrödingerlike picture, namely, the picture where we observe the mo- tion of an ensemble of trajectories rather than that of a single trajectory, ordinary diffusion is produced by a dynamical operator proportional to a second-order spatial derivative. Again, a condition of ordinary differentiability is ensured. In conclusion, the existence of a time-scale separation between microscopic dynamics and the experimental observation, usually made at the macroscopic level, ensures that the results of the observation process can be predicted by means of theoretical prescriptions based on ordinary mathematical procedures resting on the differentiability assumption.

A totally different condition is generated if the time-scale separation is not adequate. We have separately discussed the Heisenberg and Schrödinger pictures. From the results of Sec. III, devoted to the Heisenberg picture, we see that when the condition of time-scale separation is insured, the ordinary Van Hove method can be applied, and standard exponential relaxation follows. In the absence of the condition of timescale separation, the method of Van Hove must be generalized, and this, in turn, yields a generalization of the exponential relaxation, a condition that according to Glöckle and Nonnenmacher $[12,13]$ turns out to be very efficient for describing such nonstandard physical processes as polymer dynamics.

From within the Schrödinger picture, discussed in Sec. IV, the lack of a time-scale separation, and the consequent requirement for a generalization of the Van Hove method, yields the striking replacement of the ordinary Laplacian operator with the fractional Laplacian discussed in Appendix A. We have to point out that even in this case randomness has a very subtle origin, implying a departure from the differentiable condition of Hamiltonian dynamics, and the replacement of the continuous variable responsible for microscopic fluctuations with a dichotomous variable. This process, changing a two-state physical dynamics, compatible with a Hamiltonian picture, into a nondifferentiable process is clearly described by the sketch of Fig. 1. We firmly restate here that the replacement of a continuous variable picture with a discrete representation is also done in the case of ordinary statistical mechanics, where this way of forcing randomness within differentiable dynamics does not imply a departure from differentiable dynamics at a statistical level. When the condition of time-scale separation is not available, the Van Hove procedure of forcing microscopic dynamics to become random has a striking manifestation at the macroscopic level under the form of the fractional Laplacian of Appendix A.

To stress the main conclusion of the paper, we set aside the non-Hamiltonian cases, and especially those where stochastic processes are assumed to be already at work, whatever their origin might be. This is where the microscopic dynamics are genuinely not differentiable, and whether or not this lack of microscopic differentiability has macroscopic manifestations depends on whether or not this nondifferentiability can be transmitted to the macroscopic level as a result of memory. The present paper also sheds light on this interesting, but less fundamental, issue. A much more important condition is that where the dynamics are described by a Hamiltonian. In this case the microscopic dynamics are differentiable, and in principle, there would be no microscopic randomness to transmit to the macroscopic level.

However, one of the main tenets of the current literature 
on the derivation of statistical mechanics from dynamics rests on the conviction that randomness can be generated from Hamiltonian dynamics either as a consequence of the action of infinitely many degrees of freedom or as a consequence of chaos. The Hamiltonian of Eq. (7) is an example of the first kind, and the sense of the Van Hove limit in that case is that the very short and the very large time scales are beyond the range of observation. Thus, randomness is a consequence of the observer's limitations. The physical condition illustrated by Fig. 1, conversely, might be related in some way to the case of chaos, in a sense that it is convenient to properly discuss here.

Let us consider the well-known case of a map resulting in intermittency [31], for instance, that introduced by Geisel and Thomae [32]. The motion under study is characterized by a long permanence in conditions of regular motion with bursts of chaotic dynamics concerning the transition from one laminar region to the other. This is not a genuinely Hamiltonian dynamics, and the generator of intermittency is in fact a map. According to the general discussion of Ref. [31], similar properties can also be exhibited by genuinely Hamiltonian systems such as the attractive model of the eggcarton two-dimensional potential investigated by Geisel, Zacherl, and Radons [33]. Another interesting model of the same type is the three-dimensional Hamiltonian flow more recently studied by Zaslavsky, Steven, and Weitzner [34]. This paper, as well as that of Ref. [33], can be regarded as an example of Hamiltonian derivation of the Lévy processes, and, consequently, according to the point of view adopted in this paper, an example of a dynamical process described at the macroscopic level by a fractional derivative in spite of its Hamiltonian, and, consequently, differentiable nature.

It has to be stressed, however, that the theory behind this macroscopic derivation should be applied to a numerical treatment of these processes: This theory does not have anything to do with the ideally exact solution of a dynamical model resting on a continuous treatment, and the assumption of differentiability at any order. The numerical treatment is characterized by round-off errors and, more importantly, by a discrete time representation, which forces the system to depart from the conditions ideally established by its Hamiltonian property. The intermittency of these systems is sufficient to create at least temporary conditions of nondifferentiability which are then transmitted to the macroscopic level and changed, in the way described in this paper, into fractional derivatives. The generalized version of the Van Hove method serves the basic purpose of introducing conditions of microscopic nondifferentiability without leaving the theoretical treatment and without entering the level of the numerical solution. In the case of the dynamical approach to Lévy processes, the meaning of the generalized Van Hove method is closely related to the Lévy-Gnedenko generalized central limit theorem [26]. This is so because forcing the fluctuating variable to become rigorously dichotomous imposes enough coarse graining on the system so as to produce randomness. We also note that in the case 1 $<\beta<2, \quad T$ is proportional to the mean time duration the map spends in one laminar region. Consequently, if $t \gg T$, the number $N \equiv t / T$, which is very large, corresponds to the number of uncorrelated space transitions of intensity $\mid x$ $-x^{\prime} \mid$, with probability $1 /\left|x-x^{\prime}\right|^{1+\beta}$, made by the system.
The Lévy-Gnedenko theorem insures that for $N$ tending to infinity a Lévy diffusion process is generated. From a physical point of view this has the same effect as forcing $T$ to zero.

We think that at this stage we are also in the right position to establish an appealing connection with exciting results of the research work of Refs. [5,6]. Although some doubts are expressed by the authors of Ref. [6] on the role of chaos in generating Brownian motion (a role which might be sufficient but not necessary, as, in a sense, it is also shown by our chain model of Sec. II), we are inclined to believe that the connection established by the authors of Ref. [5] between Brownian motion and chaos is very attractive. However, it applies to the condition of time-scale separation, which is expected to generate ordinary statistical mechanics. This paper is devoted, on the contrary, to studying dynamical cases where this time-scale separation is missing, and consequently, nonordinary statistical mechanics is generated. This might generate the wrong impression that the method of analysis adopted in Ref. [5], based on the use of the Kolmogorov-Sinai entropy [35], cannot be applied to the dynamical systems with no time-scale separation. Actually, it has been recently shown [36] that the Kolmogorov-Sinai entropy can be generalized so as also to be made efficient in the case of fractal dynamics. Furthermore, it was also recently shown [37] that the same entropic arguments naturally lead to the same form of spatial fractional derivative as that of Eq. (59). This means that the Van Hove generalized method discussed in this paper is expected to establish a natural bridge between the nonextensive entropy of Tsallis [38] and the fractional derivative of Eq. (59), much in the same way as the ordinary Van Hove method makes Hamiltonian dynamics compatible with the standard diffusion equation of Eq. (48), and so with ordinary extensive thermodynamics behind it [39].

\section{ACKNOWLEDGMENT}

One of the authors (A.R.) thanks the INFM for partial support of this research.

\section{APPENDIX A}

We want to define the integrals and derivatives used in the fractional calculus introduced in the text. First of all, let us recall the Riemann-Liouville definition of fractional integral. Let us assume $\beta \geqslant 0$ and, following Ref. [40], let us define the $\beta$ fractional integral of the function $f(x)$ to be

$$
\frac{d^{-\beta}}{d x^{-\beta}} f(x)=\frac{1}{\Gamma(\beta)} \int_{c}^{x} \frac{f(y) d y}{(x-y)^{1-\beta}} .
$$

We also define the $\beta$ fractional derivative of the function $f(x)$ as

$$
\frac{d^{\beta}}{d x^{\beta}} f(x)=\frac{1}{\Gamma(n-\beta)} \frac{d^{n}}{d x^{n}} \int_{c}^{x} \frac{f(y) d y}{(x-y)^{\beta-n+1}},
$$

where $n$ is the smallest integer larger than $\beta$, that is, $n$ $=[\beta]+1$. The constant $c$ in the limits of the integrals is usually set to 0 (Riemann definition) or to $-\infty$ (Liouville 
definition). It is easy to show that for a $\beta$ integer both definitions reduce to the ordinary definitions of derivative and integral.

An equivalent definition makes use of Fourier transform [41]. Let us consider a function $f(x)$ with Fourier transform $\hat{f}(k)$ :

$$
\begin{gathered}
\hat{f}(k)=\frac{1}{\sqrt{2 \pi}} \int_{-\infty}^{\infty} f(x) e^{i k x} d x, \\
f(x)=\frac{1}{\sqrt{2 \pi}} \int_{-\infty}^{\infty} \hat{f}(x) e^{-i k x} d k .
\end{gathered}
$$

The $n$th derivative of $f(x)$ can be written as

$$
D^{(n)} f(x)=\mathcal{F}^{-1}\left[(-i k)^{n} \hat{f}(k) ; x\right],
$$

and a possible way of generalizing this expression to the $\beta$ derivative of $f(x)$ is

$$
D^{(\beta)} f(x)=\mathcal{F}^{-1}\left[(-i k)^{\beta} \hat{f}(k) ; x\right] .
$$

Equation (A6) is equivalent to the convolution product

$$
D^{(\beta)} f(x)=\frac{1}{\sqrt{2 \pi}} \int_{-\infty}^{\infty} d^{(\beta)}(x-y) f(y) d y,
$$

where

$$
d^{(\beta)}(x) \equiv \frac{1}{\sqrt{2 \pi}} \int_{-\infty}^{\infty}(-i k)^{\beta} e^{-i k x} d k
$$

It is possible to calculate an explicit representation of integral (A8), and the results are [41]

$$
\begin{aligned}
D_{+}^{(\beta)} f(x) & =\frac{1}{\Gamma(n-\beta)} \frac{d^{n}}{d x^{n}} \int_{-\infty}^{x} \frac{f(y) d y}{(x-y)^{\beta-n+1}}, \\
D_{-}^{(\beta)} f(x) & =-\frac{1}{\Gamma(n-\beta)} \frac{d^{n}}{d x^{n}} \int_{x}^{\infty} \frac{f(y) d y}{(x-y)^{\beta-n+1}},
\end{aligned}
$$

where again $n=[\beta]+1$, and the $+(-)$ corresponds to evaluating integral (A8) in the upper (lower) complex $k$ plane. It is apparent that Eq. (A9) coincides with the Liouville definition of the fractional derivative

$$
D_{+}^{(\beta)} f(x) \equiv \frac{d^{\beta}}{d x^{\beta}} f(x) .
$$

A different way of generalizing Eq. (A5) is the following. Let us start from the definition of second derivative

$$
D^{(2)} f(x)=\mathcal{F}^{-1}\left[\left(-k^{2}\right) \hat{f}(k) ; x\right],
$$

and let us generalize it as

$$
\mathcal{D}^{(2 \beta)} f(x)=\mathcal{F}^{-1}\left[\left(-k^{2}\right)^{\beta} \hat{f}(k) ; x\right] .
$$

Equation (A13) is equivalent to the convolution product

$$
\mathcal{D}^{(2 \beta)} f(x)=\frac{1}{\sqrt{2 \pi}} \int_{-\infty}^{\infty} c^{(\beta)}(x-y) f(y) d y,
$$

where

$$
c^{(\beta)}(x) \equiv \frac{(-1)^{\beta}}{\sqrt{2 \pi}} \int_{-\infty}^{\infty}|k|^{2 \beta} e^{-i k x} d k .
$$

If

$$
0<\beta<1,
$$

integral (A15) can be evaluated explicitly [25]:

$$
c^{(\beta)}(x)=\frac{(-1)^{\beta}}{\sqrt{2 / \pi} \Gamma(-2 \beta) \cos (\beta \pi)} \frac{1}{|x|^{2 \beta+1}},
$$

and setting $2 \beta=\mu(\Rightarrow 0<\mu<2)$, we obtain

$$
\frac{1}{|x|^{\mu+1}}=-\frac{2 b(-1)^{\mu / 2}}{\mu(\mu-1) Q} c^{(\mu)}(x) .
$$

Now using Eqs. (54), (A14), and (A18), Eq. (53) can be expressed in terms of the fractional derivative $\mathcal{D}^{(\mu)}$. This gives Eq. (59). Notice that Eq. (59) is consistent with Eq. (56), since

$$
\mathcal{F}\left[c^{(\mu)}(x) ; k\right]=\left(-k^{2}\right)^{\mu / 2}=i^{\mu}|k|^{\mu} .
$$

\section{APPENDIX B}

Our aim is now to show that the conventional Van Hove limit produces, in the case of exponential relaxation for the correlation function, the same result as the traditional approach. Let us consider the following equation of motion for the probability density $p(x, t)$ :

$$
\frac{\partial}{\partial t} p(x, t)=\left\langle v^{2}\right\rangle_{\text {eq }} \int_{0}^{t} \Phi_{v}\left(t-t^{\prime}\right) \frac{\partial^{2}}{\partial x^{2}} p\left(x, t^{\prime}\right) d t^{\prime} .
$$

Equation (B1) is the same as Eq. (40) and the bath variable, now denoted with $v$, is not dichotomous. Assuming

$$
\Phi_{v}(t)=e^{-\gamma t}, \quad \gamma=\frac{1}{\tau}
$$

and differentiating both sides of Eq. (B1), we obtain

$$
\frac{\partial^{2}}{\partial t^{2}} p(x, t)=-\gamma \frac{\partial}{\partial t} p(x, t)+\left\langle v^{2}\right\rangle_{\mathrm{eq}} \frac{\partial^{2}}{\partial x^{2}} p(x, t),
$$

whose Fourier transform is given by

$$
\frac{\partial^{2}}{\partial t^{2}} \hat{p}(k, t)+\gamma \frac{\partial}{\partial t} \hat{p}(k, t)+\left\langle v^{2}\right\rangle_{\mathrm{eq}} k^{2} \hat{p}(k, t)=0 .
$$

Both Eqs. (B3) and (B4) correspond to the telegraphic equation. Considering the condition

$$
\dot{\hat{p}}(k, 0)=0,
$$


the exact solution of (B4) is written as

$$
\hat{p}(k, t)=A\left[e^{\alpha_{+}(k) t}-\frac{\alpha_{+}(k)}{\alpha_{-}(k)} e^{\alpha_{-}(k) t}\right],
$$

with $A$ to be specified according to the normalization condition, and $\alpha_{ \pm}$given by

$$
\alpha_{ \pm}=-\frac{\gamma}{2} \pm \frac{\gamma}{2} \sqrt{1-\frac{4\left\langle v^{2}\right\rangle_{\mathrm{eq}} k^{2}}{\gamma^{2}}} .
$$

Assuming now

$$
\frac{4\left\langle v^{2}\right\rangle_{\mathrm{eq}} k^{2}}{\gamma^{2}} \ll 1
$$

solution (B6) can be expanded in a Taylor series, giving

$$
\begin{aligned}
\hat{p}(k, t)= & A\left[\exp \left(-\frac{\left\langle v^{2}\right\rangle_{\mathrm{eq}} k^{2}}{\gamma} t\right)-\frac{\left\langle v^{2}\right\rangle_{\mathrm{eq}} k^{2} / \gamma}{\gamma-\left\langle v^{2}\right\rangle_{\mathrm{eq}} k^{2} / \gamma}\right. \\
& \left.\times \exp \left(-\gamma t+\frac{\left\langle v^{2}\right\rangle_{\mathrm{eq}} k^{2}}{\gamma} t\right)+O\left(\frac{\left\langle v^{2}\right\rangle_{\mathrm{eq}}^{2}}{\gamma^{3}}\right)\right] .
\end{aligned}
$$

Note that setting condition (B8) is compatible with the Van Hove limit resting on setting $\gamma \rightarrow \infty$. At this stage we have to apply to Eq. (B9) the Van Hove limit, which in addition to setting $\gamma \rightarrow \infty$ also rests on making the limit $\left\langle v^{2}\right\rangle_{\mathrm{eq}} \rightarrow \infty$ in such a way that

$$
D=\operatorname{Lim}_{\text {VH }} \frac{\left\langle v^{2}\right\rangle_{\mathrm{eq}}}{\gamma}
$$

We note that this procedure makes the terms $O\left(\left\langle v^{2}\right\rangle_{\mathrm{eq}}^{2} / \gamma^{3}\right)$ disappear, so as to recover the ordinary diffusion equation solution

$$
\hat{p}(k, t)=A e^{-D k^{2} t}
$$

On the other hand, the same result can be obtained by applying the Van Hove limit directly to the equation of motion (B1) with the assumption (B2), and also making use of both Eqs. (42) and (45). This proves that ordinary Brownian diffusion rests on the Van Hove limit.
[1] L. Van Hove, Physica (Amsterdam) 21, 517 (1955).

[2] R. W. Zwanzig, in Lectures in Theoretical Physics, Boulder 1960 (Interscience, New York, 1961), Vol. III, p. 6.

[3] In our opinion the meaning of the Van Hove method has been made transparent by the discussion of Zwanzig in Ref. [2]. For this reason, we refer ourselves to the work of Zwanzig more than to the original paper by Van Hove (Ref. [1]).

[4] T. Y. Petrosky and H. Hasegawa, Physica A 160, 351 (1989).

[5] P. Gaspard, M. E. Brigs, M. K. Francis, J. V. Sengers, R. W. Gammon, J. R. Dorfman, and R. V. Calabrese, Nature (London) 394, 865 (1998).

[6] D. Dürr and H. Spohn, Nature (London) 394, 831 (1998).

[7] P. Allegrini, P. Grigolini, and B. J. West, Phys. Rev. E 54, 4760 (1996).

[8] V. Seshadri and B. J. West, Proc. Natl. Acad. Sci. USA 79, 4501 (1982).

[9] B. J. West and P. Grigolini, in Applications of Fractional Calculus in Physics, edited by R. Hilfer (World Scientific, Singapore, 1998).

[10] H. C. Fogedby, Phys. Rev. Lett. 73, 2517 (1994).

[11] R. N. Mantegna and H. E. Stanley, Phys. Rev. Lett. 73, 2946 (1994).

[12] W. G. Glöckle and T. F. Nonnenmacher, J. Stat. Phys. 71, 741 (1993).

[13] W. G. Glöckle and T. F. Nonnenmacher, Rheol. Acta 33, 337 (1994).

[14] P. Grigolini, Adv. Chem. Phys. 62, 1 (1985).

[15] L. Fonda, G. C. Ghirardi, and A. Rimini, Rep. Prog. Phys. 41, 587 (1978)

[16] M. H. Lee, Phys. Rev. Lett. 51, 1227 (1983).

[17] R. F. Fox, J. Math. Phys. 18, 2331 (1977).
[18] D. Vitali and P. Grigolini, Phys. Rev. A 39, 1486 (1989).

[19] H. Mori, Prog. Theor. Phys. 33, 423 (1965).

[20] H. Mori, Prog. Theor. Phys. 34, 399 (1965).

[21] P. Grigolini, G. Grosso, G. Pastori-Parravicini, and M. Sparpaglione, Phys. Rev. B 27, 7342 (1983).

[22] W. G. Glöckle and T. F. Nonnenmacher, Macromolecules 24, 6426 (1991).

[23] P. Grigolini, in Noise in Nonlinear Dynamical Systems, edited by F. Moss and P. V. E. McClintock (Cambridge University Press, Cambridge, 1989), Chap. 5, p. 161.

[24] G. Trefan, E. Floriani, B. J. West, and P. Grigolini, Phys. Rev. E 50, 2564 (1994).

[25] I. S. Gradshteyn and I. M. Ryzhik, Table of Integrals, Series, and Products (Academic, New York, 1994).

[26] B. V. Gnedenko and A. N. Kolmogorov, Limit Distributions for Sums of Independent Random Variables (Addison-Wesley, Reading, MA, 1954).

[27] R. Metzler and T. F. Nonnenmacher, Phys. Rev. E 57, 6409 (1998).

[28] R. Metzler, J. Klafter, and I. M. Sokolov, Phys. Rev. E 58, 1621 (1998).

[29] G. Zumofen and J. Klafter, Phys. Rev. E 47, 851 (1993).

[30] It is important to stress that the fractional time derivative adopted in Ref. [28] aims at a purpose different from that of this section. Conversely, the fractional time derivative adopted in Ref. [27], equivalent to setting $T=0$ in the denominator of $T^{\beta} /\left(T^{2}+t^{\prime}\right)^{\beta / 2}$ in Eq. (43), conflicts with the generalized Van Hove method and consequently with the numerical and theoretical conclusions of both Refs. [29] and [7].

[31] J. Klafter, G. Zumofen, and M. F. Shlesinger, in Chaos-The Interplay Between Stochastic and Deterministic Behaviour, 
Lecture Notes in Physics Vol. 457 (Springer, New York, 1995).

[32] T. Geisel and S. Thomae, Phys. Rev. Lett. 52, 1936 (1984).

[33] T. Geisel, A. Zacherl, and G. Radons, Z. Phys. B 71, 117 (1988).

[34] G. M. Zaslavsky, D. Stevens, and H. Weitzner, Phys. Rev. E 48, 1683 (1993).

[35] A. N. Kolmogorov, Dokl. Acad. Sci. SSSR 119, 861 (1958); Ya. G. Sinai, ibid. 124, 768 (1959).
[36] C. Tsallis, A. R. Plastino, and W.-M. Zheng, Chaos Solitons Fractals 8, 885 (1997).

[37] M. Buiatti, P. Grigolini, and A. Montagnini, preprint, cond-mat/9809108.

[38] C. Tsallis, J. Stat. Phys. 52, 479 (1988).

[39] C. Tsallis, Physica A 221, 277 (1995).

[40] Fractional Calculus and its Applications, edited by B. Ross (Springer-Verlag, Berlin, 1975).

[41] P. Závada, e-print funct-an/9608002. 